\title{
Perineural Dexamethasone to Improve Postoperative Analgesia with Peripheral Nerve Blocks: A Meta-Analysis of Randomized Controlled Trials
}

\author{
Gildasio S. De Oliveira Jr., Lucas J. Castro Alves, Autoun Nader, \\ Mark C. Kendall, Rohit Rahangdale, and Robert J. McCarthy \\ Department of Anesthesiology, Feinberg School of Medicine, Northwestern University, 241 East Huron F5-704, \\ Chicago, IL 60611, USA \\ Correspondence should be addressed to Gildasio S. De Oliveira Jr.; g-jr@northwestern.edu
}

Received 30 June 2014; Accepted 9 October 2014; Published 18 November 2014

Academic Editor: Stefan Evers

Copyright (C) 2014 Gildasio S. De Oliveira Jr. et al. This is an open access article distributed under the Creative Commons Attribution License, which permits unrestricted use, distribution, and reproduction in any medium, provided the original work is properly cited.

\begin{abstract}
Background. The overall effect of perineural dexamethasone on postoperative analgesia outcomes has yet to be quantified. The main objective of this quantitative review was to evaluate the effect of perineural dexamethasone as a nerve block adjunct on postoperative analgesia outcomes. Methods. A systematic search was performed to identify randomized controlled trials that evaluated the effects of perineural dexamethasone as a block adjunct on postoperative pain outcomes in patients receiving regional anesthesia. Metaanalysis was performed using a random-effect model. Results. Nine randomized trials with 760 subjects were included. The weighted mean difference ( $99 \%$ CI) of the combined effects favored perineural dexamethasone over control for analgesia duration, 473 (264 to 682) minutes, and motor block duration, 500 (154 to 846) minutes. Postoperative opioid consumption was also reduced in the perineural dexamethasone group compared to control, $-8.5(-12.3$ to -4.6$) \mathrm{mg}$ of IV morphine equivalents. No significant neurological symptoms could have been attributed to the use of perineural dexamethasone. Conclusions. Perineural dexamethasone improves postoperative pain outcomes when given as an adjunct to brachial plexus blocks. There were no reports of persistent nerve injury attributed to perineural administration of the drug.
\end{abstract}

\section{Introduction}

Regional anesthesia has been commonly used as a strategy to mitigate postoperative pain in surgical patients [1-3]. Peripheral nerve blocks are particularly important in the ambulatory surgical setting since patients in that setting do not have access to potent intravenous analgesics in order to manage postoperative pain $[4,5]$. However, a major limitation of peripheral nerve blocks can be a limited duration of analgesia $[6,7]$. In order to circumvent that limitation, peripheral nerve catheters that offer continuously delivery of local anesthetics have been proposed as an efficient method of postoperative analgesia $[8,9]$. Nevertheless, peripheral nerve catheters are costly and can be cumbersome to manage in the outpatient surgical setting $[10,11]$.

Dexamethasone is a systemic glucocorticoid commonly used to reduce postoperative nausea/vomiting pain and to improve quality of recovery after surgery [12-14]. Recently, several studies have examined the use of perineural dexamethasone in order to prolong analgesic duration of peripheral nerve blocks with variable results. However, the aggregated effect of perineural dexamethasone on analgesia outcomes has yet to be quantified. In addition, the safety of perineural dexamethasone also needs to be further examined [15].

The main objective of the current investigation was to evaluate the effect of perineural dexamethasone on analgesia outcomes along with peripheral nerve blocks. We also sought to examine safety concerns related to the use of perineural dexamethasone.

\section{Methods}

This quantitative systematic review was conducted following the guidelines of the PRISMA statement [16]. 
2.1. Systematic Search. Published reports of randomized trials evaluating the effects of perineural dexamethasone on postoperative anesthesia/analgesia were searched using the National Library of Medicine's PubMed database, Embase, the Cochrane Database of Systematic Reviews, and Google Scholar inclusive to August 15, 2013. Free text and MeSH terms "dexamethasone," "nerve," "block," "regional," "surgery," "analgesia," and "anesthesia” were used individually and in various pairwise combinations. No language restriction was used. An attempt to identify additional studies not found by the primary search methods was made by reviewing the reference lists from identified studies. No search was performed for unpublished studies. This initial search yielded 164 studies.

2.2. Selection of Included Studies. The study's inclusion and exclusion criteria were established before the systematic search. Two authors (Gildasio S. De Oliveira and Lucas J. Castro Alves) independently evaluated the abstract and results of the articles obtained by the initial search. Articles that were clearly not relevant based on our inclusion and exclusion criteria were excluded at this phase. Disagreements on inclusion of the articles were resolved by discussion among the evaluators. If an agreement could not be reached, the dispute was resolved with the help of a third investigator (Autoun Nader).

2.3. Inclusion and Exclusion Criteria. We included randomized controlled trials that examined perineural administration of dexamethasone for peripheral nerve blocks with the perineural administration of an inactive (placebo) control group. Trials reporting on the effects of dexamethasone on neuraxial blocks were excluded. Trials evaluating the effect of perineural dexamethasone in animals were also excluded. Studies containing the concurrent use of perineural drug were excluded if a direct comparison of dexamethasone and control could not be established. Volunteer studies in which subjects did not undergo surgical procedures were also excluded. Included studies had to report on clinical outcomes such as duration and/or onset of anesthesia/analgesia. No minimum sample size was required for inclusion in the metaanalysis.

2.4. Validity Scoring. Two authors (Gildasio S. De Oliveira and Lucas J. Castro Alves) independently read the included reports and assessed their methodological validity using a modified Jadad five-point quality scale [17]. The scale evaluates the study for the following: randomization, double blind evaluation, concealment of study group to evaluator, valid randomization method, and completeness of data at follow-up. As only randomized trials were included in the analysis, the minimum possible score of an included trial was 1 and the maximum was 5. Trials were not excluded or weighted in the analysis based on quality assessment scores.

2.5. Data Extraction. Two authors (Gildasio S. De Oliveira and Lucas J. Castro Alves) independently evaluated the full manuscripts of all included trials and performed data extraction using a data collection form specifically developed for this quantitative review.

Data extracted from trials included perineural dexamethasone dose, sample size, number of subjects in treatment groups, type of procedure, type of regional block, volume and dose of local anesthetics, analgesia duration (minutes), motor block onset and duration, cumulative opioid consumption, late pain (24 hours), and adverse events related to the regional anesthesia technique. Postoperative opioid consumption was converted to the equivalent dose of intravenous morphine [18]. Visual analog scale or numeric rating scale of pain was converted to a $0-10$ numeric rating scale.

Data were initially extracted from tables. For data not available in tables, the data were abstracted from available figures. Dichotomous data on the presence or absence of adverse effects were extracted and converted to incidence while continuous data was recorded using mean and standard deviation. Data presented only as median and range was converted to means and standard deviation using previously described methodology [19]. The most conservative value was used when the same outcome was reported more than one time for a determined period.

\subsection{Definition of Relevant Outcome Data}

2.6.1. Primary Outcomes. These included analgesia time (time in minutes to the first request of analgesia and/or reported pain), cumulative opioid consumption, early pain ( $<4$ hours), and late pain (24 hours).

2.6.2. Secondary Outcomes. These included oOnset and duration of motor block, onset of sensory block, and nerve block complications.

2.7. Meta-Analyses. The weighted mean differences (WMD) with 99\% confidence interval (CI) were determined and reported for continuous data. For dichotomous data (adverse effects), the Peto odds ratio (to account for the potential of zero counts in the cells for low frequency outcomes) and $99 \%$ CI are reported. A significant effect compared to control required that the $99 \%$ CI for continuous data did not include zero and, for dichotomous data, the confidence interval did not include 1.0. Due to the inclusion of different procedures, we used a random effects model in an attempt to generalize our findings to studies not included in our meta-analysis. Although more computationally intensive, the random effects model has an advantage to the fixed effect model since it does not rely on the assumption that a true effect size is exactly the same in all combined studies [20]. Publication bias was evaluated by examining asymmetric funnel plots using Egger's regression test [21, 22]. A one sided $P<0.05$ was considered as an indication of an asymmetric funnel plot. A file drawer analysis described by Rosenthal was performed in the case of an asymmetric funnel plot [23]. The test estimates the lowest number of additional studies that if they would become available would reduce the combined effect to nonsignificance assuming the average $z$-value of the combined $P$ values of these missing studies would be 0 . 
Heterogeneity of the included studies was considered to be present if the $I^{2}$ statistic was greater than $30 \%$. Further analysis was planned a priori to explore relevant heterogeneity. Subgroup analysis was performed to investigate the effect of type of regional block and local anesthetic on the effects of examined outcomes. A $Q$ statistic was used to compare the effects between subgroups. The proportion of the total variance explained by the covariates $\left(R^{2}\right)$ was calculated by dividing random effects pooled estimates of variance (Tau squared) within studies by total variance (total Tau squared). The value obtained was then subtracted from 1 . When values fall outside the range of 0 to $100 \%$, they were set to the closest value $(0 \%$ or $100 \%)$.

A metaregression analysis was performed to evaluate the presence of a linear association between the perineural dexamethasone dose and the effect size on the analgesia duration [24]. Analysis was performed using Comprehensive Meta-Analysis software version 2 (Biostat, Englewood, NJ) and STATA version 11 (College Station, Texas, USA).

\section{Results}

Of the 164 initially evaluated abstracts, 20 studies were initially selected (Figure 1). Eleven studies were subsequently excluded: five studies were not randomized trials [25-29], three studies evaluated systemic dexamethasone [30-32], two studies did not evaluate peripheral blocks $[33,34]$, and one study did not provide a direct comparison [35]. The characteristics of included studies are listed in Table 1. The evaluated trials included data from 730 subjects and were published between 2006 and 2013 [36-44]. The median (IQR) number of patients in the included studies receiving perineural dexamethasone was 30 (24 to 49). The median (IQR) modified Jadad scale score was 4 (4 to 5). The trials tested the administration of perineural dexamethasone to patients undergoing upper extremity blocks for orthopedic procedures.

3.1. Analgesia Duration (the First Request for Pain Medications and/or Report of Pain in Minutes). The overall effect of seven studies (nine comparisons) [36-42] that examined perineural dexamethasone on analgesia duration compared to control favored dexamethasone with a weighted mean difference (99\% CI) of 473 (264 to 682) minutes (Figure 2). Two studies contained two independent comparisons that were included in the analysis [38, 39]. The funnel plot was asymmetric suggesting the presence of publication bias; however, Rosenthal analysis suggested that it would be required to identify 6337 missing studies in order to change the analysis. Heterogeneity was high $\left(I^{2}=93\right)$ but $22 \%$ of the total variance was explained by different blocks (interscalene versus supraclavicular or axillary). The overall effect of perineural dexamethasone on analgesia duration was increased when a subgroup analysis was performed including only interscalene blocks, WMD ( $99 \%$ CI) of 615 (528 to 701) min compared to control. There was no evidence for an asymmetric funnel plot $(P=0.48)$.

Metaregression analysis did not detect a linear association between perineural dexamethasone dose and duration of analgesia $(P=0.92)$.

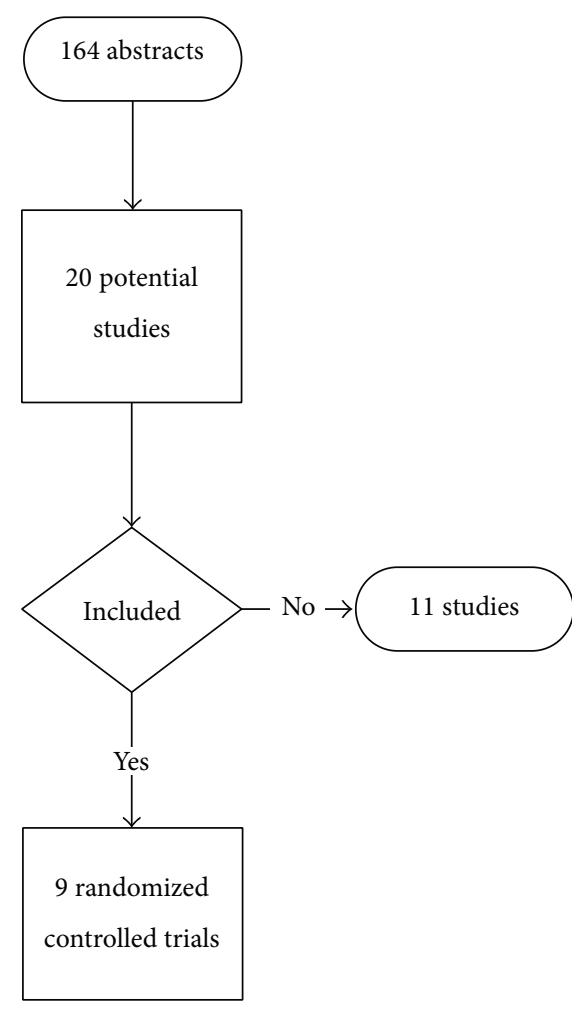

FIGURE 1: Flow chart outlining retrieved, excluded, and evaluated randomized controlled trials. Some trials evaluated multiple doses of dexamethasone.

3.2. Cumulative Opioid Consumption. The aggregated effect of four studies (six comparisons) [38-40,43] that evaluated perineural dexamethasone on opioid consumption favored perineural dexamethasone compared to control, WMD ( $99 \%$ CI) of -8.5 ( -12.3 to -4.6$) \mathrm{mg}$ of IV morphine equivalents (Figure 3). Funnel plot was asymmetric suggesting the possibility of publication bias $(P=0.04)$. Heterogeneity was high $\left(I^{2}=86\right)$ and could not be explained by the type of the regional block or local anesthetic used. In contrast, the effect of perineural dexamethasone on postoperative opioid consumption was detected when bupivacaine and/or ropivacaine was used, WMD (95\% CI) of $-10.8(-12.0$ to 9.6) $\mathrm{mg}$ of IV morphine equivalents, but not when lidocaine was used, 1.3 ( -6.8 to 9.4$) \mathrm{mg}$ of IV morphine equivalents, $P<0.001$.

3.3. Late Pain ( 24 hours). The overall effect of three studies $[40,43,44]$ examining the effect of perineural dexamethasone on late pain did not show a beneficial effect of dexamethasone compared to control, WMD (99\% CI) of $-0.03(-4.2$ to 4.1$)$. Heterogeneity was high $\left(I^{2}=96\right)$ and it could not be explained by the type of the regional block or local anesthetic used.

3.4. Early Pain ( $<4$ hours). Only one study evaluated the effect of perineural dexamethasone on early pain suggesting a minor effect of perineural dexamethasone compared to control, WMD ( $99 \% \mathrm{CI})$ of $-0.4(-0.6$ to -0.2$)$ [44]. The 


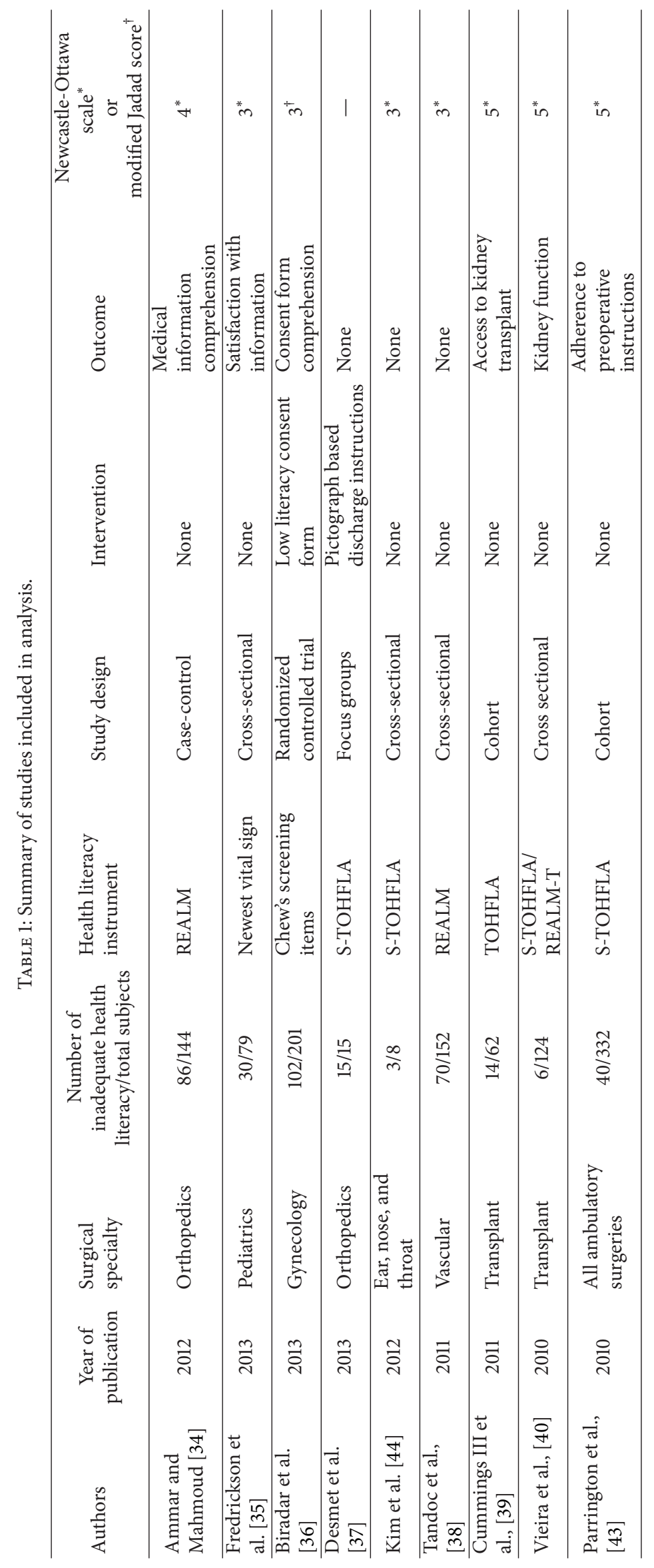




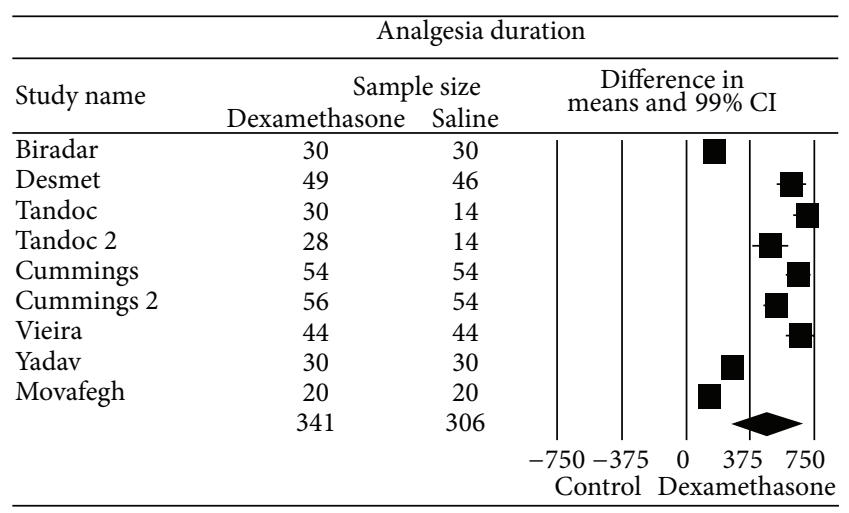

FIgURE 2: Pooled data evaluating the effect of perineural dexamethasone on analgesia duration compared to control. Data evaluated using a random effects model. Point estimate (99\% CI) for overall effect was 473 (264 to 682) minutes. Weighted mean difference for individual study represented by square on forest plot with $99 \%$ confidence interval of the difference shown as solid line. Larger sized square and thicker $99 \%$ confidence interval line denote larger sample size. The diamond represents the pooled estimate and uncertainty for the effects of perineural dexamethasone compared to control.

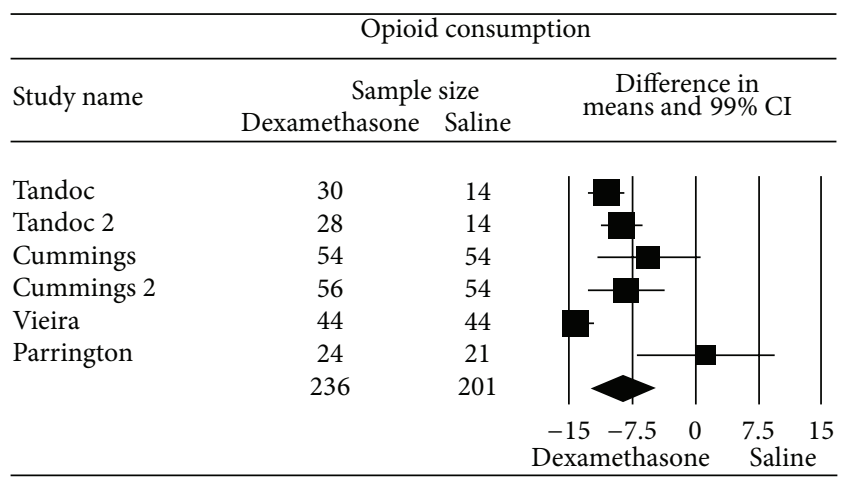

Figure 3: Pooled data evaluating the effect of perineural dexamethasone on postoperative opioid consumption compared to control. Data evaluated using a random effects model. Point estimate (99\% CI) for overall effect was $-8.5(-12.3$ to -4.6$) \mathrm{mg}$ of IV morphine equivalents. Weighted mean difference for individual study represented by square on forest plot with $99 \%$ confidence interval of the difference shown as solid line. Larger sized square and thicker $99 \%$ confidence interval line denote larger sample size. The diamond represents the pooled estimate and uncertainty for the effects of perineural dexamethasone compared to control.

study evaluated the use of perineural dexamethasone for patients receiving an interscalene brachial plexus block with levobupivacaine.

3.5. Block Onset (Sensory and Motor). The aggregated effect of four studies [36, 41-43] that examined perineural dexamethasone on sensory block onset did not demonstrate a benefit of perineural dexamethasone compared to control, WMD (99\% CI) of -0.6 ( -2.9 to 1.5$)$ min. Heterogeneity was high $\left(I^{2}=77\right)$ and it could not be explained by the type of the regional block or type of local anesthetic used.

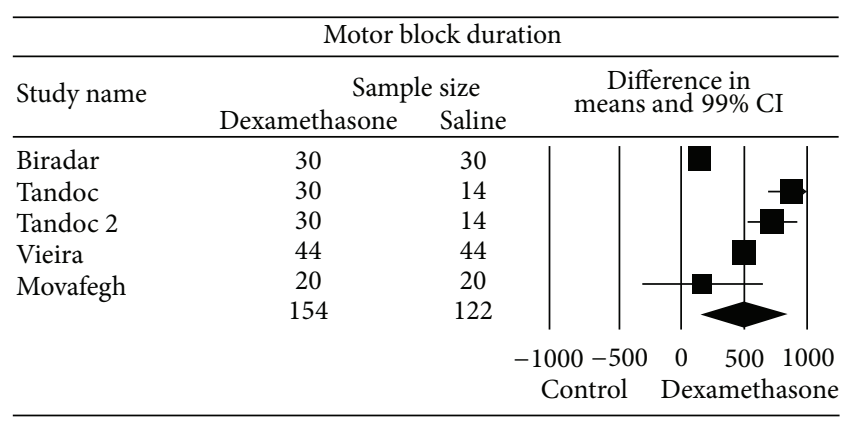

FIGURE 4: Pooled data evaluating the effect of perineural dexamethasone on motor block duration compared to control. Data evaluated using a random effects model. Point estimate (99\% CI) for overall effect was 500 (154 to 846) min. Weighted mean difference for individual study represented by square on forest plot with $99 \%$ confidence interval of the difference shown as solid line. Larger sized square and thicker $99 \%$ confidence interval line denote larger sample size. The diamond represents the pooled estimate and uncertainty for the effects of perineural dexamethasone compared to control.

The combined effect of four studies [36, 41-43] that examined the effect of perineural dexamethasone compared to control on the onset of motor block did not detect a benefit of perineural dexamethasone relative to a large confidence interval, WMD $(99 \% \mathrm{CI})$ of -1.0 (-3.1 to 1.1$)$. Heterogeneity was high $\left(I^{2}=77\right)$ and it could not be explained by the type of the regional block or type of local anesthetic used.

3.6. Motor Block Duration. The overall effect of four studies (five comparisons) [36, 38, 40, 42] evaluating perineural dexamethasone compared to control on motor block duration favored dexamethasone, WMD (99\% CI) of 500 (154 to 846) min (Figure 4). One study had two independent comparisons that were included in the analysis [38]. Egger's regression did not suggest the presence of an asymmetric funnel plot $(P=0.1)$. Heterogeneity was high $\left(I^{2}=98\right)$ but it could be largely explained (64\%) by studies that evaluated the effect of perineural dexamethasone on axillary/supraclavicular blocks as opposed to the interscalene block. The effect of perineural dexamethasone on motor block duration was greater for blocks performed with bupivacaine and/or ropivacaine, WMD (95\% CI) of 696 (378 to 1015) compared to lidocaine, WMD (95\% CI) of 155 (128 to 181).

3.6.1. Safety Analysis. The majority of studies did not report any complications related to the use of perineural dexamethasone on peripheral nerve block. One study reported one episode of hypoesthesia in the dexamethasone group 4 months after surgery and none in the control group [37]. One study reported a greater but not statistically significant difference of tingling/numbness in the dexamethasone group (40\%) compared to the saline group 26(\%) two weeks after the operation.

\section{Discussion}

The most important finding of the current investigation was the substantially longer duration of analgesia in patients 
receiving dexamethasone as adjunct to brachial plexus blocks compared to control. Specifically for the interscalene approach, the use of dexamethasone with local anesthetics (ropivacaine or bupivacaine) prolonged analgesia duration for over 10 hours. In addition, the longer analgesic duration was accompanied by a lower consumption of postoperative opioids. Side effects were transient and could not be specifically attributed to the use of perineural dexamethasone. Taken together, our results suggest that perineural dexamethasone is an efficacious strategy to improve postoperative analgesia in subjects receiving brachial plexus blocks.

Our results are clinically important because the use of perineural dexamethasone as an adjunct to interscalene blocks provided analgesia duration for 24 hours in patients undergoing orthopedic surgery. Single injection nerve block with local anesthetics and dexamethasone may provide comparable analgesic results as peripheral nerve catheters for the first 24 hours after surgical procedures [9, 45, 46]. However, peripheral nerve catheters can have greater costs and complications such as catheter dislodgment or inadequate tip placement which can minimize the analgesic benefit of peripheral nerve catheters $[8,47,48]$. Nevertheless, future studies comparing single shot blocks using local anesthetics with dexamethasone to peripheral nerve catheters on analgesia outcomes are needed.

It was interesting to note the lack of benefit of perineural dexamethasone on late pain (24 hours) scores. Several reasons may explain this finding. First, it is possible that the similar pain scores in both groups were achieved due to a greater consumption of opioids in the control group compared to the perineural dexamethasone. Second, the duration of analgesia lasted approximately twenty four hours and the benefit may have decreased when the late pain outcome was measured. Lastly, the number of studies was not sufficient to detect an effect despite the apparent negative aggregate effect on late pain (WMD of -0.03).

We were unable to detect an association between perineural dexamethasone dose and effect size on the evaluated outcomes using metaregression analysis. It is possible that lower doses of dexamethasone may provide similar results on postoperative analgesia outcomes as the most commonly used $8 \mathrm{mg}$ dexamethasone dose. In fact, a recent study suggested that even lower doses such as $1 \mathrm{mg}$ may provide similar results on analgesia duration as greater dexamethasone doses [49].

We did not detect significant long lasting nerve complications that could be attributed to the use of perineural dexamethasone in our study population. One study reported one case of hypoesthesia in the dexamethasone group but the subject also had spinal disc herniation at the level of C45 which could have explained the symptoms [37]. Another study reported an increased but not statistically significant difference in the incidence of numbness and tingling in the dexamethasone group fourteen days after surgery. Since basic science studies have suggested the possibility of nerve toxicity by different perineural adjuncts, it is important that future studies provide longer follow-up evaluations of the included subjects [50-52].
It is also important to note that two recent studies have demonstrated similar benefits on analgesic duration of systemic compared to perineural dexamethasone for upper and lower extremity blocks $[35,37]$. A potential advantage of perineural dexamethasone is the avoidance of undesirable side effects associated with the use of systemic dexamethasone [53-56]. Future studies evaluating the use of perineural dexamethasone on analgesia outcomes would benefit from the inclusion of a comparison group to evaluate the systemic dexamethasone administration.

Despite a benefit on postoperative analgesic duration and postoperative opioid consumption, the included studies did not evaluate the beneficial effect of perineural dexamethasone on more global recovery parameters $[57,58]$. Inclusion of more global recovery outcomes is important since analgesic interventions may or may not demonstrate a benefit when more global recovery outcomes are evaluated [59-62]. Few studies have, in fact, been able to demonstrate a beneficial effect of regional anesthesia techniques on patient centered outcomes [63].

Our current quantitative review should only be interpreted in the context of its limitations. Some of our primary analysis revealed an asymmetric funnel plot suggesting the possibility of publication bias. Based on a Rosenthal analysis it is unlikely that the results would be changed by the detection of unpublished negative studies but the overall effect could be possibly reduced. In addition, publication bias was not detected when we performed a subgroup analysis of a single type of brachial plexus block. We also observed significant heterogeneity that could be partially explained by type of brachial plexus block and type of local anesthetic used. Lastly, we were unable to quantitatively estimate a potential harmful effect of perineural dexamethasone on postoperative nerve symptoms due to the low number of studies reporting those outcomes $(N<3)$.

In summary, perineural dexamethasone seems to improve analgesia duration and decrease opioid consumption when used as an adjunct to brachial plexus blocks. For interscalene blocks, perineural dexamethasone prolonged analgesia for more than ten hours. The included studies did not report on persistent nerve injury that could be attributed to the use of perineural dexamethasone. Perineural dexamethasone should be considered to improve postoperative analgesia in subjects receiving brachial plexus blocks for surgical procedures.

\section{Conflict of Interests}

The authors declare that there is no conflict of interests regarding the publication of this paper.

\section{Authors' Contribution}

Gildasio S. De Oliveira helped in study design, study conduct, data analysis, and paper preparation. Lucas J. Castro Alves contributed to study design, study conduct, and paper preparation. Autoun Nader helped in study design, study conduct, and paper preparation. Mark C. Kendall contributed to paper preparation. Rohit Rahangdale helped in study design and 
paper preparation. Robert J. McCarthy contributed to study design, data analysis, and paper preparation.

\section{Acknowledgment}

The authors acknowledge Department of Anesthesiology, Feinberg School of Medicine, Northwestern University.

\section{References}

[1] Z. M. Naja, F. M. Ziade, M. A. El-Rajab, N. Naccash, and J.M. Ayoubi, "Guided paravertebral blocks with versus without clonidine for women undergoing breast surgery: a prospective double-blinded randomized study," Anesthesia and Analgesia, vol. 117, no. 1, pp. 252-258, 2013.

[2] N. Kumar, S. Shashni, R. Singh, and A. Jain, "Mandibular nerve block for peri-operative pain relief using a peripheral nerve stimulator," Anaesthesia, vol. 67, no. 1, pp. 77-78, 2012.

[3] M. R. Guilfoyle, A. Helmy, D. Duane, and P. J. A. Hutchinson, "Regional scalp block for postcraniotomy analgesia: a systematic review and meta-analysis," Anesthesia and Analgesia, vol. 116, no. 5, pp. 1093-1102, 2013.

[4] G. Danelli, S. Bonarelli, A. Togn et al., "Prospective randomized comparison of ultrasound-guided and neurostimulation techniques for continuous interscalene brachial plexus block in patients undergoing coracoacromial ligament repair," British Journal of Anaesthesia, vol. 108, no. 6, pp. 1006-1010, 2012.

[5] L. F. R. Falcão, M. V. Perez, I. de Castro et al., "Minimum effective volume of $0.5 \%$ bupivacaine with epinephrine in ultrasound-guided interscalene brachial plexus block," British Journal of Anaesthesia, vol. 110, no. 3, pp. 450-455, 2013.

[6] M. P. M. Rancourt, N. T. Albert, M. Côté, D. R. Létourneau, and P. M. Bernard, "Posterior tibial nerve sensory blockade duration prolonged by adding dexmedetomidine to ropivacaine," Anesthesia and Analgesia, vol. 115, no. 4, pp. 958-962, 2012.

[7] D. Marhofer, S. C. Kettner, P. Marhofer, S. Pils, M. Weber, and M. Zeitlinger, "Dexmedetomidine as an adjuvant to ropivacaine prolongs peripheral nerve block: a volunteer study," British Journal of Anaesthesia, vol. 110, no. 3, pp. 438-442, 2013.

[8] B. M. Ilfeld, V. J. Loland, N. S. Sandhu et al., "Continuous femoral nerve blocks: the impact of catheter tip location relative to the femoral nerve (anterior versus posterior) on quadriceps weakness and cutaneous sensory block," Anesthesia \& Analgesia, vol. 115, no. 3, pp. 721-727, 2012.

[9] A. Brammar and N. Sharma, "Interscalene catheter safety: a novel technique," Anaesthesia, vol. 68, no. 2, pp. 210-211, 2013.

[10] B. Yanovski, L. Gaitini, D. Volodarski, and B. Ben-David, "Catastrophic complication of an interscalene catheter for continuous peripheral nerve block analgesia," Anaesthesia, vol. 67, no. 10, pp. 1166-1169, 2012.

[11] O. Choquet and X. Capdevila, "Three-dimensional highresolution ultrasound-guided nerve blocks: a new panoramic vision of local anesthetic spread and perineural catheter tip location," Anesthesia and Analgesia, vol. 116, no. 5, pp. 1176-1181, 2013.

[12] G. S. de Oliveira, L. J. S. Castro-Alves, S. Ahmad, M. C. Kendall, and R. J. McCarthy, "Dexamethasone to prevent postoperative nausea and vomiting: an updated meta-analysis of randomized controlled trials," Anesthesia and Analgesia, vol. 116, no. 1, pp. 58-74, 2013.
[13] N. H. Waldron, C. A. Jones, T. J. Gan, T. K. Allen, and A. S. Habib, "Impact of perioperative dexamethasone on postoperative analgesia and side-effects: systematic review and metaanalysis," British Journal of Anaesthesia, vol. 110, no. 2, pp. 191200, 2013.

[14] G. S. de Oliveira, S. Ahmad, P. C. Fitzgerald et al., "Dose ranging study on the effect of preoperative dexamethasone on postoperative quality of recovery and opioid consumption after ambulatory gynaecological surgery," British Journal of Anaesthesia, vol. 107, no. 3, pp. 362-371, 2011.

[15] E. Yilmaz-Rastoder, M. S. Gold, K. A. Hough, G. F. Gebhart, and B. A. Williams, "Effect of adjuvant drugs on the action of local anesthetics in isolated rat sciatic nerves," Regional Anesthesia and Pain Medicine, vol. 37, no. 4, pp. 403-409, 2012.

[16] A. Liberati, D. G. Altman, J. Tetzlaff et al., “The PRISMA statement for reporting systematic reviews and meta-analyses of studies that evaluate health care interventions: explanation and elaboration," Journal of Clinical Epidemiology, vol. 62, no. 10, pp. el-e34, 2009.

[17] A. R. Jadad, R. A. Moore, D. Carroll et al., "Assessing the quality of reports of randomized clinical trials: is blinding necessary?" Controlled Clinical Trials, vol. 17, no. 1, pp. 1-12, 1996.

[18] P. E. Macintyre and L. B. Ready, "Pharmacology of opioids," in Acute Pain Management: A Practical Guide, pp. 15-49, WB Saunders, 2nd edition, 2001.

[19] S. P. Hozo, B. Djulbegovic, and I. Hozo, "Estimating the mean and variance from the median, range, and the size of a sample," BMC Medical Research Methodology, vol. 5, article 13, 2005.

[20] R. DerSimonian and N. Laird, "Meta-analysis in clinical trials," Controlled Clinical Trials, vol. 7, no. 3, pp. 177-188, 1986.

[21] M. Egger, G. D. Smith, M. Schneider, and C. Minder, "Bias in meta-analysis detected by a simple, graphical test," British Medical Journal, vol. 315, no. 7109, pp. 629-634, 1997.

[22] G. S. de Oliveira Jr., R. Chang, M. C. Kendall, P. C. Fitzgerald, and R. J. McCarthy, "Publication bias in the anesthesiology literature," Anesthesia \& Analgesia, vol. 114, no. 5, pp. 1042-1048, 2012.

[23] R. Rosenthal, "The file drawer problem and tolerance for null results," Psychological Bulletin, vol. 86, no. 3, pp. 638-641, 1979.

[24] G. S. De Oliveira Jr., B. Balliu, A. Nader, and R. J. McCarthy, "Dose-ranging effects of intrathecal epinephrine on anesthesia/analgesia: a meta-analysis and metaregression of randomized controlled trials," Regional Anesthesia and Pain Medicine, vol. 37, no. 4, pp. 423-432, 2012.

[25] N. S. Cooper, "Intravenous dexamethasone and caudal analgesia," British Journal of Anaesthesia, vol. 106, no. 1, pp. 145-146, 2011.

[26] N. de la Fuente and F. R. Altermatt, "Adding dexamethasone to peripheral nerve blocks can give better postoperative analgesia," British Journal of Anaesthesia, vol. 108, no. 1, p. 161, 2012.

[27] K. D. Candido, A. Germanovich, R. F. Ghaly, G. H. Gorelick, and N. N. Knezevic, "Case report: computed tomography scanguided gasserian ganglion injection of dexamethasone and lidocaine for the treatment of recalcitrant pain associated with herpes simplex type 1 infection of the ophthalmic division of the trigeminal nerve," Anesthesia and Analgesia, vol. 112, no. 1, pp. 224-227, 2011.

[28] M. J. Meyer, E. J. Krane, K. R. Goldschneider, and N. J. Klein, "Neurological complications associated with epidural analgesia in children: a report of 4 cases of ambiguous etiologies," Anesthesia \& Analgesia, vol. 115, no. 6, pp. 1365-1370, 2012. 
[29] D. Falzon, P. Jefferson, and D. R. Ball, "Dexamathasone and ondansetron in paediatric tonsillectomy," Anaesthesia, vol. 67, no. 2, pp. 191-192, 2012.

[30] B. B. Abdelmalak, A. M. Bonilla, D. Yang et al., "The hyperglycemic response to major noncardiac surgery and the added effect of steroid administration in patients with and without diabetes," Anesthesia and Analgesia, vol. 116, no. 5, pp. 1116-1122, 2013.

[31] V. Hermans, F. de Pooter, F. de Groote, S. de Hert, and P. van der Linden, "Effect of dexamethasone on nausea, vomiting, and pain in paediatric tonsillectomy," British Journal of Anaesthesia, vol. 109, no. 3, pp. 427-431, 2012.

[32] M. T. Aouad, V. G. Nasr, V. G. Yazbeck-Karam et al., "A comparison between dexamethasone and methylprednisolone for vomiting prophylaxis after tonsillectomy in inpatient children: a randomized trial," Anesthesia and Analgesia, vol. 115, no. 4, pp. 913-920, 2012.

[33] F. M. Ahadian, K. McGreevy, and G. Schulteis, "Lumbar transforaminal epidural dexamethasone: a prospective, randomized, double-blind, dose-response trial," Regional Anesthesia and Pain Medicine, vol. 36, no. 6, pp. 572-578, 2011.

[34] A. S. Ammar and K. M. Mahmoud, "Effect of adding dexamethasone to bupivacaine on transversus abdominis plane block for abdominal hysterectomy: a prospective randomized controlled trial," Saudi Journal of Anaesthesia, vol. 6, no. 3, pp. 229-233, 2012.

[35] M. J. F. Fredrickson, T. K. Danesh-Clough, and R. White, "Adjuvant dexamethasone for bupivacaine sciatic and ankle blocks: results from 2 randomized placebo-controlled trials," Regional Anesthesia and Pain Medicine, vol. 38, no. 4, pp. 300307, 2013.

[36] P. A. Biradar, P. Kaimar, and K. Gopalakrishna, "Effect of dexamethasone added to lidocaine in supraclavicular brachial plexus block: a prospective, randomised, double-blind study," Indian Journal of Anaesthesia, vol. 57, no. 2, pp. 180-184, 2013.

[37] M. Desmet, H. Braems, M. Reynvoet et al., "I.V. and perineural dexamethasone are equivalent in increasing the analgesic duration of a single-shot interscalene block with ropivacaine for shoulder surgery: a prospective, randomized, placebocontrolled study," British Journal of Anaesthesia, vol. 111, no. 3, pp. 445-452, 2013.

[38] M. N. Tandoc, L. Fan, S. Kolesnikov, A. Kruglov, and N. D. Nader, "Adjuvant dexamethasone with bupivacaine prolongs the duration of interscalene block: a prospective randomized trial," Journal of Anesthesia, vol. 25, no. 5, pp. 704-709, 2011.

[39] K. C. Cummings III, D. E. Napierkowski, I. Parra-Sanchez et al., "Effect of dexamethasone on the duration of interscalene nerve blocks with ropivacaine or bupivacaine," British Journal of Anaesthesia, vol. 107, no. 3, pp. 446-453, 2011.

[40] P. A. Vieira, I. Pulai, G. C. Tsao, P. Manikantan, B. Kelle, and N. R. Connelly, "Dexamethasone with bupivacaine increases duration of analgesia in ultrasound-guided interscalene brachial plexus blockade," European Journal of Anaesthesiology, vol. 27, no. 3, pp. 285-288, 2010.

[41] R. K. Yadav, B. P. Sah, P. Kumar, and S. N. Singh, "Effectiveness of addition of neostigmine or dexamethasone to local anaesthetic in providing perioperative analgesia for brachial plexus block: a prospective, randomized, double blinded, controlled study," Kathmandu University Medical Journal, vol. 6, no. 23, pp. 302309, 2008.

[42] A. Movafegh, M. Razazian, F. Hajimaohamadi, and A. Meysamie, "Dexamethasone added to lidocaine prolongs axillary brachial plexus blockade," Anesthesia and Analgesia, vol. 102, no. 1, pp. 263-267, 2006.

[43] S. J. Parrington, D. O’Donnell, V. W. S. Chan et al., "Dexamethasone added to mepivacaine prolongs the duration of analgesia after supraclavicular brachial plexus blockade," Regional Anesthesia and Pain Medicine, vol. 35, no. 5, pp. 422-426, 2010.

[44] Y. J. Kim, G. Y. Lee, D. Y. Kim, C. H. Kim, H.-J. Baik, and S. Heo, "Dexamathasone added to levobupivacaine improves postoperative analgesia in ultrasound guided interscalene brachial plexus blockade for arthroscopic shoulder surgery," Korean Journal of Anesthesiology, vol. 62, no. 2, pp. 130-134, 2012.

[45] B. M. Ilfeld, N. S. Sandhu, V. J. Loland et al., "Ultrasoundguided (Needle-in-Plane) perineural catheter insertion: the effect of catheter-insertion distance on postoperative analgesia," Regional Anesthesia and Pain Medicine, vol. 36, no. 3, pp. 261$265,2011$.

[46] A. Schnabel, C. H. Meyer-Frießem, P. K. Zahn, and E. M. Pogatzki-Zahn, "Ultrasound compared with nerve stimulation guidance for peripheral nerve catheter placement: a metaAnalysis of randomized controlled trials," British Journal of Anaesthesia, vol. 111, no. 4, pp. 564-572, 2013.

[47] D. Marhofer, P. Marhofer, L. Triffterer, M. Leonhardt, M. Weber, and M. Zeitlinger, "Dislocation rates of perineural catheters: a volunteer study," British Journal of Anaesthesia, vol. 111, no. 5, pp. 800-806, 2013.

[48] B. M. Ilfeld, "Continuous peripheral nerve blocks: a review of the published evidence," Anesthesia and Analgesia, vol. 113, no. 4, pp. 904-925, 2011.

[49] J. Liu, K. A. Richman, and N. Elkassabany, "Is there a dosage effect of dexamethasone as an adjuvant for brachial plexus nerve block? A prospective randomized double-blinded clinical study," in Proceedings of the 38th Annual Regional Anesthesiology and Acute Pain Medicine Meeting, 2013, Abstract number: A067.

[50] C. M. S. Cereda, G. R. Tofoli, L. G. Maturana et al., "Local neurotoxicity and myotoxicity evaluation of cyclodextrin complexes of bupivacaine and ropivacaine," Anesthesia and Analgesia, vol. 115, no. 5, pp. 1234-1241, 2012.

[51] J. B. McAlvin, G. Reznor, S. A. Shankarappa, C. F. Stefanescu, and D. S. Kohane, "Local toxicity from local anesthetic polymeric microparticles," Anesthesia and Analgesia, vol. 116, no. 4, pp. 794-803, 2013.

[52] S. A. Shankarappa, I. Sagie, J. H. Tsui et al., "Duration and local toxicity of sciatic nerve blockade with coinjected site 1 sodium-channel blockers and quaternary lidocaine derivatives," Regional Anesthesia and Pain Medicine, vol. 37, no. 5, pp. 483489, 2012.

[53] R. Bartlett and A. J. Hartle, "Routine use of dexamethasone for postoperative nausea and vomiting: the case against," Anaesthesia, vol. 68, no. 9, pp. 892-896, 2013.

[54] K. Dhatariya and R. P. Mahajan, "II. Does dexamethasoneinduced hyperglycaemia contribute to postoperative morbidity and mortality?" British Journal of Anaesthesia, vol. 110, no. 5, pp. 674-675, 2013.

[55] S. Ali Khan, D. L. Mcdonagh, and T. J. Gan, "Wound complications with dexamethasone for postoperative nausea and vomiting prophylaxis: a moot point?" Anesthesia and Analgesia, vol. 116, no. 5, pp. 966-968, 2013.

[56] C. S. Bolac, A. H. Wallace, G. Broadwater, L. J. Havrilesky, and A. S. Habib, "The impact of postoperative nausea and vomiting prophylaxis with dexamethasone on postoperative wound complications in patients undergoing laparotomy for 
endometrial cancer," Anesthesia and Analgesia, vol. 116, no. 5, pp. 1041-1047, 2013.

[57] B. F. Gornall, P. S. Myles, C. L. Smith et al., "Measurement of quality of recovery using the QoR-40: a quantitative systematic review," British Journal of Anaesthesia, vol. 111, no. 2, pp. 161-169, 2013.

[58] S. Y. Kim, J. M. Kim, J. H. Lee, B. M. Song, and B. N. Koo, "Efficacy of intraoperative dexmedetomidine infusion on emergence agitation and quality of recovery after nasal surgery," British Journal of Anaesthesia, vol. 111, no. 2, pp. 222-228, 2013.

[59] P. W. H. Peng, C. Li, E. Farcas et al., "Use of low-dose pregabalin in patients undergoing laparoscopic cholecystectomy," British Journal of Anaesthesia, vol. 105, no. 2, pp. 155-161, 2010.

[60] G. S. de Oliveira Jr., P. Fitzgerald, L. F. Streicher, R.-J. Marcus, and R. J. McCarthy, "Systemic lidocaine to improve postoperative quality of recovery after ambulatory laparoscopic surgery," Anesthesia and Analgesia, vol. 115, no. 2, pp. 262-267, 2012.

[61] M. V. Grady, E. Mascha, D. I. Sessler, and A. Kurz, "The effect of perioperative intravenous lidocaine and ketamine on recovery after abdominal hysterectomy," Anesthesia and Analgesia, vol. 115, no. 5, pp. 1078-1084, 2012.

[62] G. S. de Oliveira, P. C. Fitzgerald, R.-J. Marcus, S. Ahmad, and R. J. McCarthy, "A dose-ranging study of the effect of transversus abdominis block on postoperative quality of recovery and analgesia after outpatient laparoscopy," Anesthesia and Analgesia, vol. 113, no. 5, pp. 1218-1225, 2011.

[63] L. J. S. Catro-Alves, V. L. F. de Azevedo, T. F. de Freitas Braga, A. C. Goncalves, and G. S. de Oliveira Jr., "The effect of neuraxial versus general anesthesia techniques on postoperative quality of recovery and analgesia after abdominal hysterectomy: a prospective, randomized, controlled trial," Anesthesia and Analgesia, vol. 113, no. 6, pp. 1480-1486, 2011. 


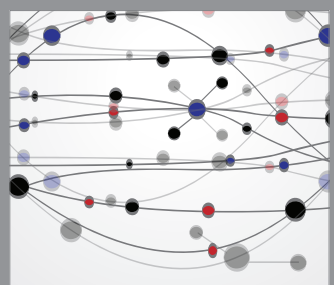

The Scientific World Journal
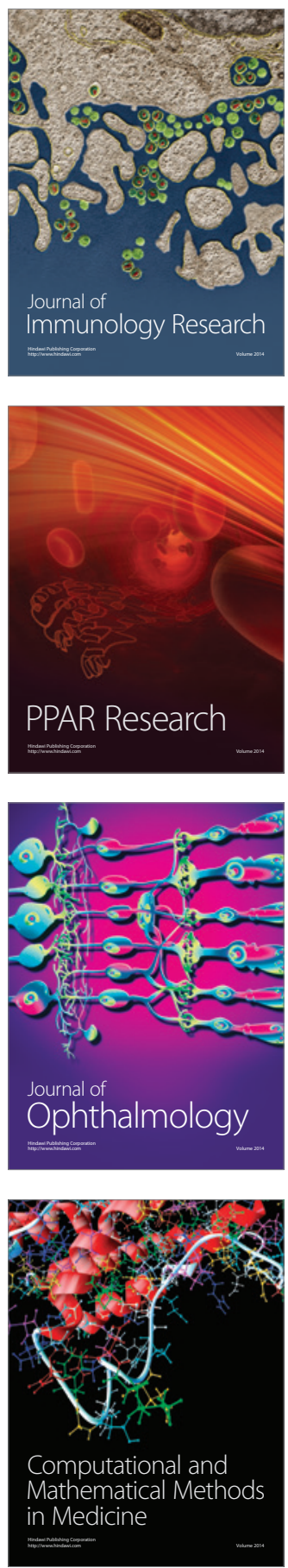

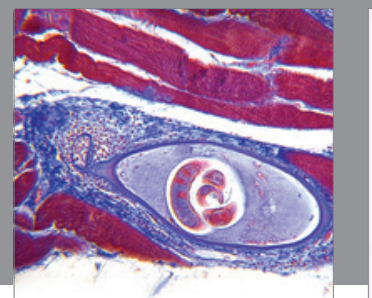

Gastroenterology

Research and Practice
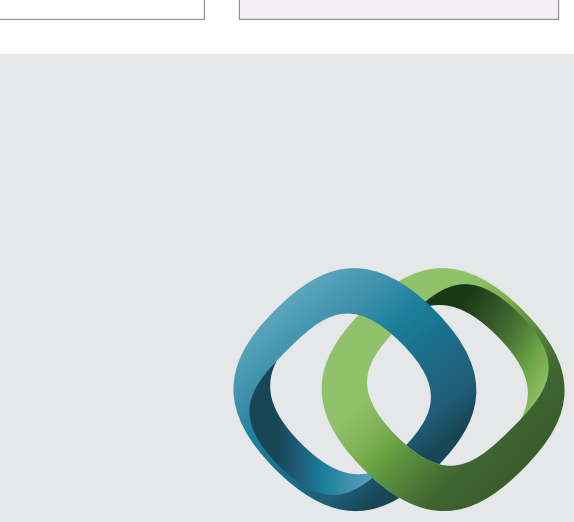

\section{Hindawi}

Submit your manuscripts at

http://www.hindawi.com
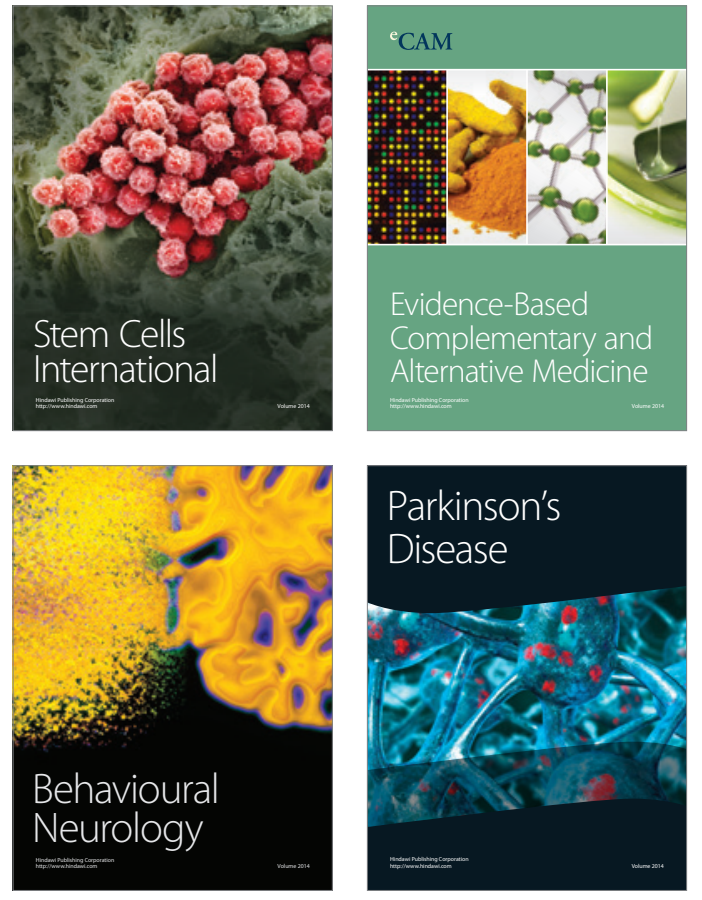
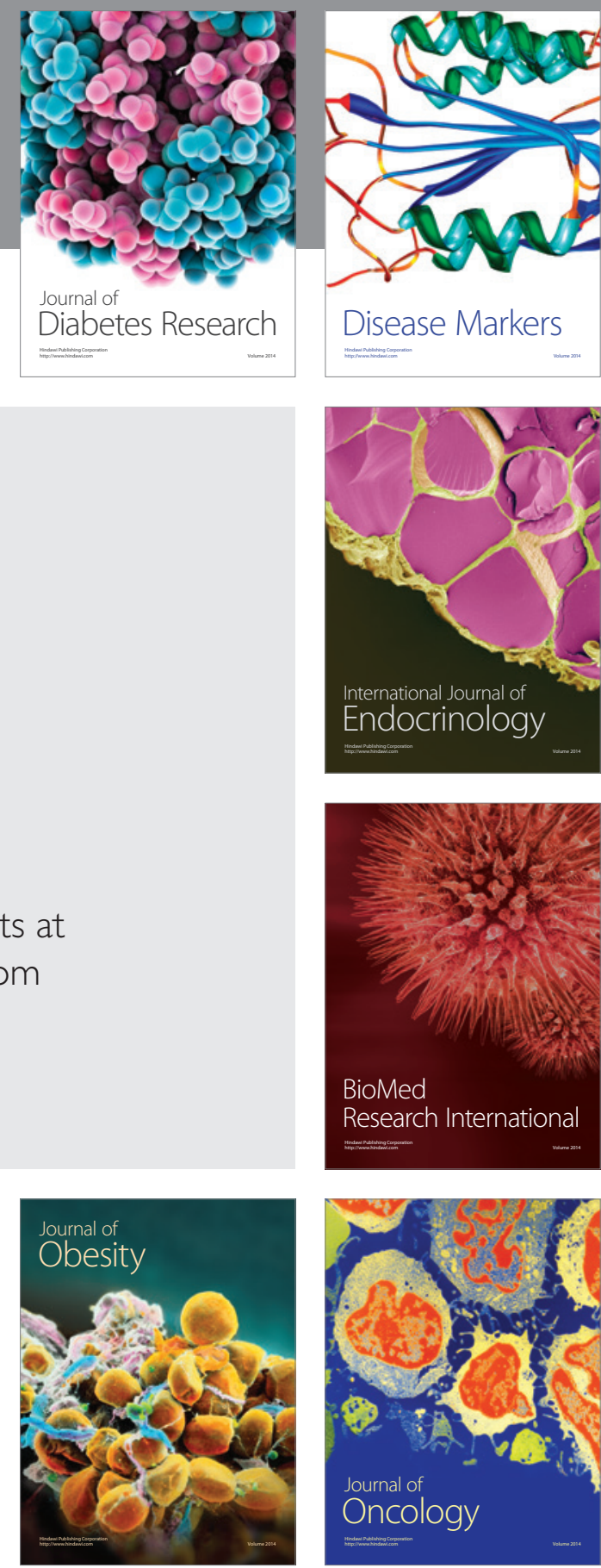

Disease Markers
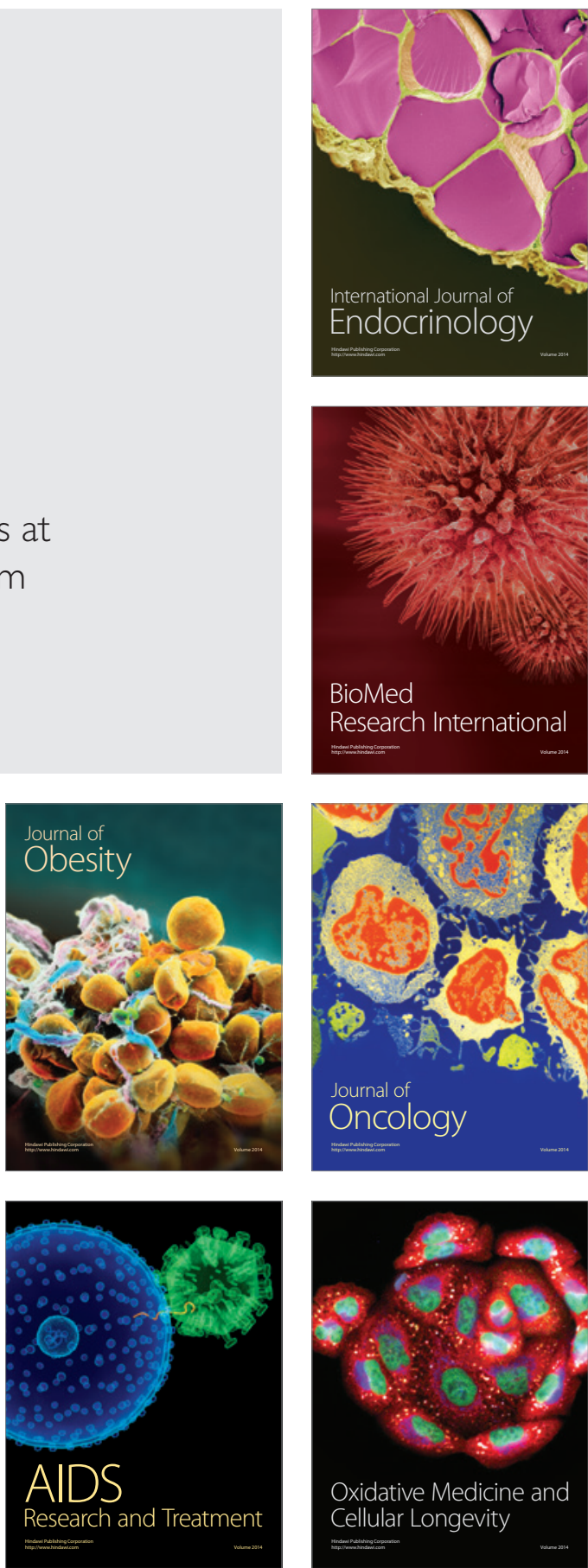